\title{
Shave of the brave: Self-concept in chemotherapy-induced hair loss
}

\author{
Sinead Power \\ School of Nursing and Midwifery, UCC
}

"A woman who cuts her hair is about to change her life"... Coco Chanel

\section{Background}

Historically, hair has played an important role in society, symbolising masculinity and virility in males and youthfulness and beauty in females. Furthermore, hair has traditionally been indicative of social, religious and professional status. For example, Christian priests and monks once shaved the crowns of their head to symbolise a lack of vanity and their vow of chastity. In ancient Egypt, the Pharaoh always wore a wig to denote his status. For women, hair is an important indicator of femininity and attractiveness in society. The term "crowning glory" was used in the Bible to denote a woman's hair: "but for a woman, if her hair is abundant, it is a glory to her, for her hair is given to her for a covering" (Corinthians 11: 15). Figures from the United States indicate that the average woman spends approximately $\$ 50,000$ on her hair over her lifetime, thus illustrating the importance of hair in society. Thus, given the significance of hair in society, it is clear that the loss of hair, particularly within the context of cancer treatment is a source of distress for individuals.

In recent years, advances in the treatment of cancer mean that many individuals are living longer with a cancer diagnosis. However, these advances in treatment mean that more patients experience the many side effects associated with cancer and its treatment. These include nausea and vomiting, fatigue, memory loss; neuropathic side effects such as pins and needles; and hair loss. While all of these side effects impact on the lives of individuals, hair loss resulting from chemotherapy is a side effect which causes significant disruption to patients' daily lives. This is mainly due to the fact that hair plays an important role in one's outward presentation and appearance. Consequently, the loss of one's hair impacts on one's physical, psychological and social well-being. Chemotherapy-induced hair loss affects individuals (male and female) with a variety of cancers including breast, testicular, lung, gynecological and hematological cancers. For example, hair loss resulting from chemotherapy occurs in 50\% of patients with breast cancer. Research studies have illustrated that some individuals may refuse potentially curative treatment due to anxiety and concern relating to hair loss. There is a perception that chemotherapy-induced hair loss is mainly associated with the loss of head hair. Patients may not always be aware that hair 
loss affects all areas of the body. This includes the loss of eyelashes, eyebrows, nasal hair, pubic hair, chest hair and leg hair. Chemotherapy-induced hair loss is temporary. For many individuals, hair generally begins to return between 6-9 months post the commencement of chemotherapy. However, for a number of patients, "new hair" is often very different to how it looked prior to hair loss. For example, hair that was once long and blonde may return grey and short. This change may have a lasting impact on an individual's selfconcept, impacting negatively on body image, perceptions of how one is viewed by others, social functioning, psychological well-being and self-worth. Due to the temporary nature of chemotherapy-induced hair loss, there is evidence that some healthcare professionals tend to under-estimate the impact of hair loss on patients. However, patients are clearly adversely affected by this side effect of cancer.

\section{Aim of the study}

The overall aim of the study was to explore and examine self-concept in patients with primary cancer who had experienced chemotherapy-induced hair loss. Primary cancer is defined as cancer which has not spread to other parts of the body. The study was conducted in two phases. In the first phase of the study, participants were interviewed regarding their experience of chemotherapy-induced hair loss. Phase 2 involved the completion of an online questionnaire. For the purposes of this paper, I will present the findings of phase 1 , in which participants were interviewed regarding their experiences of hair loss.

\section{Methods}

Permission to conduct the study was granted by the local ethics committee. Males and females were invited to take part. Flyers and posters advertising the study were distributed in a local cancer support house and a centre of excellence in cancer care. Thirteen females who had experienced chemotherapy-induced hair loss agreed to take part in the study. No males expressed an interest in taking part in the study. These participants were interviewed individually, using semi-structured interviews and were asked to speak about their experience of hair loss. These interviews were transcribed word for word by the researcher. The transcripts of the interviews were analysed in detail. Six categories emerged following analysis of the data.

\section{Findings}

Six categories emerged following the analysis of the data. These included: Preparing for hair loss; hair loss as a symbol of cancer; camouflaging hair loss; impact of hair loss on aspects of the self; physical discomfort associated with hair loss and the re-growth of hair. All participants spoke of how important it is to be adequately prepared and informed prior to the onset of hair loss. Being adequately prepared was seen as a means of "maintaining 
control", as for many patients cancer is typically associated with a loss of control. Participants were adamant that they did not want to wait until hair began to fall out, thus hair was cut short or shaved prior to hair loss.

For all participants, hair loss was perceived as a "physical label" which identified them to others as cancer patients. Some participants did not feel that they had cancer until they looked at themselves in the mirror. They described seeing themselves without hair and suddenly realising the gravity of their diagnosis. All of the women interviewed spoke of the difficulties they experienced while trying to keep their cancer diagnosis private from members of the public. They felt uncomfortable when members of the public began speaking to them about their cancer, particularly when they had not disclosed it.

All participants considered camouflaging their hair loss important. Camouflaging was seen as "essential' and "necessary" in allowing the women to continue with their day-today activities and also to "hide" their cancer diagnosis from others. The selection of the wig was identified as a key time, but a "lonely" time for participants. While the wig was considered to be a necessity, all of the women voiced their distaste for the wig, stating that they "hated" it. Wigs were also described as "hot" "tight" and "uncomfortable".

It is clear that hair loss impacted on all aspects of participants' self-concept. All of the women interviewed spoke about how their hair loss adversely affected their physical selfconcept (body image), emotional self-concept (psychological well-being) and social selfconcept (how one feels he or she is perceived by others). Participants spoke of how hair loss dramatically changed their appearance. This alteration in appearance impacted on how they felt they were viewed by others. Additionally, it is clear that hair loss caused distress to participants, thus affecting their emotional well-being.

With regard to physical self-concept (body image), one woman poignantly described herself without hair as "a medical experiment gone wrong". Furthermore, responses from other participants suggest that one's body image continues to be affected, even following the re-growth of hair. The lasting effect of hair loss on body image is vividly described by participant 12, who was one year post hair loss at the time of her interview.

\footnotetext{
"I'm still trying to find my new identity... I know the old me is gone... now I need to settle with the new me... but I'm not sure if I'm ready for that yet.... you know when I go for a shower... that's not me that I see, I have to get used to, you know get used to the new me and how I look now....." (Participant 12).
}

Hair loss also adversely impacted on the social self-concept of individuals. Participants were concerned about how others viewed them during the course of their hair loss and felt that they had a social obligation to prevent causing distress to others. The women were unsure of how to react when others complimented them on their "new hairstyle" (wig). They were also fearful of causing upset to others on seeing them wearing their bandana or on seeing their "bald head". 
All of the women interviewed spoke of how their hair loss had affected their emotional self-concept. It is clear that hair loss caused upset and distress for participants and their close family members. Participants spoke of how they were "baffled" at how their hair loss had upset them, describing it as "horrific", "desperate" and "awful". Some of the participants were mothers of young children. The response below from participant 10, poignantly describes the emotional impact of hair loss on children.

"[...] and then one morning you wake up and your hair... your hair is falling out $[\ldots]$ you know, on the pillow, in the shower [...] IT WAS ON THE KIDS [...] I hugged one of my daughters and it was all over her [...] a big clump on her shoulder [...] I mean, I took it off her as quickly as I saw it but,. [...] she saw it $[\ldots]$ she was very brave in front of me $[\ldots]$ but later on that night $[\ldots]$ she was crying in bed [...] (Participant 10).

This young mother had experienced chemotherapy-induced hair loss resulting from breast cancer 12 months prior to her interview. This poignant statement illustrates the impact that chemotherapy-induced hair loss had on this participant and her daughter.

For 12 of the participants, hair had started to return or had fully returned. One participant was 2 months post-hair loss, thus she had complete hair loss at the time of her interview. All participants were concerned about the following issues: the length of time to re-growth (one participant referred to "being in limbo" in relation to the re-growth of her hair); some participants were worried that hair may not come back at all; apprehension regarding the re-growth of hair i.e. what would it look like; the difference in quality, texture and colour of "new hair" and the readjustment associated with the re-growth of hair.

It was interesting to note that some participants had adjusted to how they looked with the wig and were reluctant to stop wearing it, once hair had started to return. The women spoke about how "comfortable" they had become wearing the wig and the apprehension they felt about revealing their "new hair" (which now looked very different to how it had looked previously).

\section{Conclusion and recommendations}

The findings of this study are significant and have important implications for cancer care. All participants spoke vividly and emotionally about their experiences of hair loss. The key categories included: preparing for hair loss; hair loss as a symbol of cancer; camouflaging hair loss; impact of hair loss on aspects of the self; physical discomfort associated with hair loss and the re-growth of hair. It is essential that healthcare professionals assist patients in their preparation for hair loss by providing them with information on what to expect and on methods to camouflage hair loss. The emotional impact of hair loss on patients and on close family members cannot be ignored and patients need to be educated on how to speak to young children about hair loss. Furthermore the physical effects of hair loss need to be incorporated into patient education material. Patients also need to 
know what to expect in relation to the re-growth of hair. Particular information needs to be given on when hair re-growth will occur and also information on the colour and texture of "new hair". Programmes such as the Look Good Feel Better Programme® should be made readily available to patients in an effort to enhance their physical, emotional and social self-concept at all stages of their hair loss. This may in turn improve how individuals deal with their hair loss. The researcher acknowledges that all participants were females. Through targeting organisations which deal specifically with males with cancer, the researcher hopes that the gender imbalance seen in phase 1 will be addressed. 